\title{
Nível de conhecimento de estudantes do curso de graduação em Odontologia sobre cigarros eletrônicos
}

\author{
Eduarda Coelho Guckert*; Caroline Zimmermann**; Maria Inês Meurer*** \\ * Cirurgiã-dentista graduada pela Universidade Federal de \\ Santa Catarina \\ ** Doutora em Odontologia (Diagnóstico Bucal) pela \\ Universidade Federal de Santa Catarina \\ *** Professora Associada, Departamento de Patologia da \\ Universidade Federal de Santa Catarina
}

Recebido: 21/05/2020. Aprovado: 23/02/2021.

\begin{abstract}
RESUMO
O cigarro eletrônico vem despontando como uma opção ao cigarro convencional, sendo considerado por alguns como uma alternativa para quem deseja parar de fumar tabaco. Muitos jovens também têm usado o dispositivo em busca de novas experiências, sensações e diversão. O objetivo desse estudo foi avaliar o nível de conhecimento dos alunos do Curso de Graduação em Odontologia da Universidade Federal de Santa Catarina sobre os cigarros eletrônicos, com base em um questionário. Também foram coletadas informações sobre o interesse para experimentar o dispositivo, a abordagem do assunto durante o curso de graduação e a autopercepção sobre o preparo para orientar pacientes sobre este assunto. O questionário foi aplicado em abril e maio de 2019 a 209 estudantes maiores de 18 anos, que foram divididos em 3 grupos: ingressantes $\left(1^{\mathrm{a}}\right.$ e $2^{\mathrm{a}}$ fases do curso), intermediários $\left(4^{\mathrm{a}} \mathrm{e}\right.$ $5^{\mathrm{a}}$ fases) e concluintes ( $9^{\mathrm{a}}$ e $10^{\mathrm{a}}$ fases). A análise descritiva foi realizada em todas as questões, e na estatística analítica assumiu-se o nível de significância de 95\%. A análise dos resultados demonstrou que estudantes concluintes possuem mais conhecimento sobre o cigarro eletrônico que ingressantes ou intermediários. No entanto, o nível de conhecimento foi considerado insatisfatório, pois mesmo entre os concluintes apenas cerca de $40 \%$ alcançaram o conceito considerado satisfatório (e que corresponderia a pelo menos $60 \%$ de acertos). A maioria dos estudantes relatou não ter tido contato com o tema durante o Curso de Graduação. Da mesma forma, a maioria dos concluintes declarou não se sentir preparada para orientar pacientes sobre cigarros eletrônicos. Os resultados deste estudo apontam para a necessidade de o currículo contemplar este conteúdo na formação dos futuros cirurgiões-dentistas, para capacitá-los a orientar seus pacientes sobre riscos e benefícios da utilização dos cigarros eletrônicos.
\end{abstract}

Descritores: Sistemas Eletrônicos de Liberação de Nicotina. Conhecimento, Educação em Odontologia.

\section{INTRODUÇÃO}

O tabagismo é reconhecido como uma doença crônica associada à dependência de nicotina, constando da Classificação Internacional de Doenças (CID-10) como um grupo de transtornos mentais e comportamentais 
devidos ao uso de fumo (CID F17). Apesar dos reconhecidos riscos à saúde relacionados a este hábito, o tabagismo permanece sendo um grande problema de saúde pública no Brasil ${ }^{1}$.

O cigarro eletrônico (CE) é um dispositivo que surgiu como uma opção ao cigarro convencional, e tem sido visto como uma alternativa para quem deseja parar de fumar tabaco; porém, a sua verdadeira eficácia para esta finalidade ainda não está comprovada ${ }^{2-7}$. Muitos jovens estão fazendo o uso desse dispositivo na busca por novas experiências, sensações e diversão ${ }^{8-11}$. Usuários de CE não se consideram fumantes, e se autointitulam vapers ${ }^{12}$.

Apesar de destinados a entregar nicotina na forma de aerossol, os CE podem ser comercializados também sem nicotina ${ }^{9}$. O número de usuários de $\mathrm{CE}$ vem aumentando a cada ano, e levantamentos mostram a existência de pelo menos 500 marcas diferentes no mercado, com considerável variabilidade no design do produto ${ }^{13,14}$. No Brasil, a comercialização, importação e propaganda de CE está proibida, assim como de quaisquer acessórios e refis a eles destinados ${ }^{15,16}$; porém, há evidências de sua comercialização de forma ilegal $^{16}$.

Sendo uma novidade, é provável que não haja conhecimento, por parte dos usuários, sobre eventuais riscos que a sua utilização possa envolver ${ }^{6,13,17-19}$. Também os profissionais de saúde precisam estar atualizados sobre o assunto e preparados para orientar seus pacientes com relação ao uso desse dispositivo. Seria desejável, portanto, que a formação desses profissionais pudesse abranger informações sobre o equipamento e seus acessórios, o conteúdo dos seus refis, os possíveis riscos à saúde decorrentes da sua utilização, e sua eficácia enquanto estratégia para cessação do tabagismo. Um cirurgião-dentista com essas informações pode orientar e solucionar dúvidas dos pacientes e, assim, atuar na promoção de saúde e prevenção de doenças, sendo a mais importante delas o câncer bucal.

Considerando este contexto, o objetivo desta pesquisa foi avaliar o nível de conhecimento dos estudantes do Curso de Graduação em Odontologia da Universidade Federal de Santa Catarina sobre o CE, traçando uma curva de conhecimento entre estudantes iniciantes, intermediários e concluintes do curso. Além disso, foi levantada a percepção dos estudantes quanto: (a) informações recebidas sobre o assunto durante o curso, (b) importância do cirurgião-dentista conhecer essas informações e (c) a autopercepção sobre o preparo pessoal para orientar um paciente sobre o uso de CE.

\section{METODOLOGIA}

$\mathrm{O}$ projeto que deu origem a esta pesquisa foi submetido ao Comitê de Ética em Pesquisa com Seres Humanos, tendo sido aprovado sob o parecer $\mathrm{n}^{\circ}$ 3.232.604. Estudantes com idade superior a 18 anos e regularmente matriculados na $1^{\mathrm{a}}$., $2^{\mathrm{a}}$., $4^{\mathrm{a}}$., $5^{\mathrm{a}}$., $9^{\mathrm{a}}$. e $10^{\mathrm{a}}$. fases do curso foram convidados a participar.

$\mathrm{Na}$ ausência de um instrumento validado na língua portuguesa, foi elaborado um questionário (quadro 1) com base em trabalhos previamente publicados $^{16,20}$. Os dados coletados foram organizados em planilhas e os participantes divididos em três grupos: $\mathrm{G} 1$ - ingressantes $\left(1^{\mathrm{a}} \mathrm{e}\right.$ $2^{\mathrm{a}}$ fases do curso); G2 - intermediários $\left(4^{\mathrm{a}}\right.$ e $5^{\mathrm{a}}$ fases); e G3 - concluintes ( $9^{\mathrm{a}}$ e $10^{\mathrm{a}}$ fases). A segmentação nestes grupos foi realizada para permitir traçar a curva de conhecimento entre estudantes iniciantes, intermediários (quando cursam as disciplinas de Patologia Bucal e Estomatologia, que abordam de forma mais enfática o conteúdo relacionado a câncer de boca) e concluintes. 
Quadro 1: Questionário aplicado aos participantes

\section{DADOS DEMOGRÁFICOS}

1. Idade:

2. Gênero:

3. Fase do curso: ( ) $1^{\mathrm{a}}$ ou $2^{\mathrm{a}}$

( ) $4^{\mathrm{a}}$ ou $5^{\mathrm{a}}$

( ) $9^{\mathrm{a}}$ ou $10^{\mathrm{a}}$

4. Status tabagista: ( ) Fumante ( ) Ex-fumante ( ) Nunca fumou

5. É (foi) usuário de cigarro eletrônico? ( ) Sim ( ) Não

[Se você respondeu SIM na questão 5, pule para a pergunta 10]

6. Você já ouviu falar em cigarros eletrônicos? ( ) Sim ( ) Não

7. Você tem curiosidade sobre a sensação de fumar um cigarro eletrônico?

( ) Definitivamente sim ( ) Provavelmente sim ( ) Provavelmente não ( ) Definitivamente não

8. Tem a pretensão de provar um cigarro eletrônico?
( ) Definitivamente sim ( ) Provavelmente sim
( ) Provavelmente não
( ) Definitivamente não

9. Se um(a) do(a)s seus(suas) melhores amigo(a)s lhe oferecesse um cigarro eletrônico, você provaria?
( ) Definitivamente sim
( ) Provavelmente sim
( ) Provavelmente não
( ) Definitivamente não

\section{$>$ Conhecimentos sobre cigarros eletrônicos}

10. Como você classifica o seu nível de conhecimento sobre o cigarro eletrônico?
( ) Não conheço nada
( ) Baixo
( ) Médio
( ) Alto

11. O hábito de fumar cigarro convencional é considerado um risco para a saúde. Comparativamente aos cigarros convencionais, você acredita que os cigarros eletrônicos são:

( ) Mais prejudiciais （） Igualmente prejudiciais （ ) Menos prejudiciais （ ) Não sei

Responda verdadeiro ou falso

12. É permitido usar cigarros eletrônicos onde o cigarro convencional é proibido.
( ) Verdadeiro ( ) Falso
( ) Não sei

13. O uso de cigarros eletrônicos pode ajudar as pessoas a parar de fumar cigarros convencionais.
( ) Verdadeiro ( ) Falso
( ) Não sei

14. Cigarros eletrônicos têm sabores agradáveis
( ) Verdadeiro ( ) Falso
( ) Não sei

15. Cigarros eletrônicos não geram fumo passivo
( ) Verdadeiro ( ) Falso
( ) Não sei

16. Cigarros eletrônicos são mais baratos que cigarros convencionais
( ) Verdadeiro ( ) Falso
( ) Não sei

17. Cigarros eletrônicos não possuem substâncias cancerígenas na sua composição
( ) Verdadeiro ( ) Falso
( ) Não sei

18. Cigarros eletrônicos podem conter nicotina
( ) Verdadeiro
( ) Falso
( ) Não sei

$>$ Sobre os conhecimentos recebidos durante o Curso de Odontologia sobre cigarros eletrônicos...

19. ...você recebeu alguma informação sobre os efeitos do uso do cigarro eletrônico na saúde?

( ) $\operatorname{Sim}$ ( ) Não

Se sim, indique em que momento/situação:

20. ...você teve alguma oportunidade de discutir em sala de aula os motivos pelos quais as pessoas usam cigarros eletrônicos? ( ) Sim ( ) Não

\section{> Sua opinião sobre a importância do cirurgião-dentista ter informacões sobre o cigarro eletrônico}

21. Em sua opinião, é papel do cirurgião-dentista ter conhecimentos sobre cigarros eletrônicos?

( ) $\operatorname{Sim}$ ( ) Não

22. Você, enquanto futuro(a) cirurgiã(o)-dentista, se sente preparado(a) para orientar um paciente que the questione sobre o uso do cigarro eletrônico?

( ) Sim ( ) Não

As informações coletadas foram agrupadas conforme segue: dados demográficos; curiosidade e influência de amigos no uso do CE; conhecimento sobre CE; levantamento sobre os conhecimentos 
recebidos durante o curso e percepção sobre as oportunidades para discussão do assunto; autoavaliação do nível de conhecimento, opinião sobre a importância de o cirurgião-dentista conhecer o assunto, e segurança para orientar o paciente caso lhe questione sobre o dispositivo eletrônico.

A análise descritiva foi realizada para todas as questões utilizando o programa Microsoft Office Excel $^{\circledR} 2010$ (Microsoft Corporation, Redmond, Washington, EUA). Para as questões relacionadas ao conhecimento sobre $\mathrm{CE}$, foi atribuído o escore 1 para os acertos e 0 para os erros. Para as questões que não foram respondidas ou cuja resposta foi "não sei", também se atribuiu escore zero. Posteriormente, os escores foram somados, totalizando o máximo de 7 acertos. Para a estatística analítica (comparação entre os grupos sobre as questões relacionadas ao conhecimento sobre cigarros eletrônicos) foi utilizado o programa IBM SPSS ${ }^{\circledR}$ versão 23 (IBM, Armonk, New York, EUA), assumindo nível de significância de $95 \%$.

\section{RESULTADOS}

A coleta de dados foi efetuada em abril e maio de 2019. Participaram da pesquisa 209 estudantes, sendo 84 do G1, 77 do G2 e 48 do G3. A média de idade dos participantes do G1 foi de 20 anos, no G2 de 22 anos e no G3 de 24 anos. A Tabela 1 mostra o número e porcentagem de participantes de cada grupo em relação ao gênero e hábito de fumar.

Tabela 1 - Distribuição das respostas dos participantes, de acordo com os grupos, segundo fatores demográficos e status tabagista

\begin{tabular}{llccccccc}
\hline \multirow{2}{*}{ Variável } & Categoria & \multicolumn{2}{c}{ G1 } & \multicolumn{2}{c}{ G2 } & \multicolumn{2}{c}{ G3 } \\
& & n & \% & n & \% & n & \% \\
\hline Gênero & Masculino & 26 & 31 & 23 & 29,9 & 16 & 33,3 \\
& Feminino & 58 & 69 & 54 & 70,1 & 32 & 66,7 \\
& Tabagista & 16 & 19 & 1 & 1,3 & 1 & 2,1 \\
\multirow{3}{*}{ Status Tabagista } & Ex-tabagista & 7 & 8,3 & 5 & 6,5 & 1 & 2,1 \\
& Não-tabagista & 57 & 67,9 & 71 & 92,2 & 46 & 95,8 \\
& Não respondeu & 4 & 4,8 & 0 & 0 & 0 & 0 \\
\hline
\end{tabular}

Quando questionados sobre o uso de CE, $20,2 \%$ do G1 afirmou fazer ou ter feito uso do dispositivo, assim como $2,6 \%$ do $\mathrm{G} 2$ e $12,5 \%$ do G3. Para aqueles que afirmaram nunca ter feito uso do CE, foram feitas perguntas adicionais relacionadas ao conhecimento sobre a existência do dispositivo, curiosidade e intenção de provar o mesmo, bem como o peso do fator social envolvido na possibilidade de testar o dispositivo. A maioria dos estudantes, nos três grupos, afirmou já ter ouvido falar do dispositivo $(\mathrm{G} 1=86,6 \%$; $\mathrm{G} 2=86,7 \%$ e $\mathrm{G} 3=$ $88 \%)$.

A metade daqueles que nunca fizeram uso do $\mathrm{CE}$ afirmou que definitivamente não tem curiosidade sobre a sensação de usar o dispositivo (50\%) nem pretensão de provar (50\%) (figura 1). No entanto, no caso de um dos melhores amigos oferecer, a taxa de recusa cai para $38 \%$, com $28 \%$ afirmando que provavelmente não usaria, e $24 \%$ que provavelmente usaria (figura 1). 

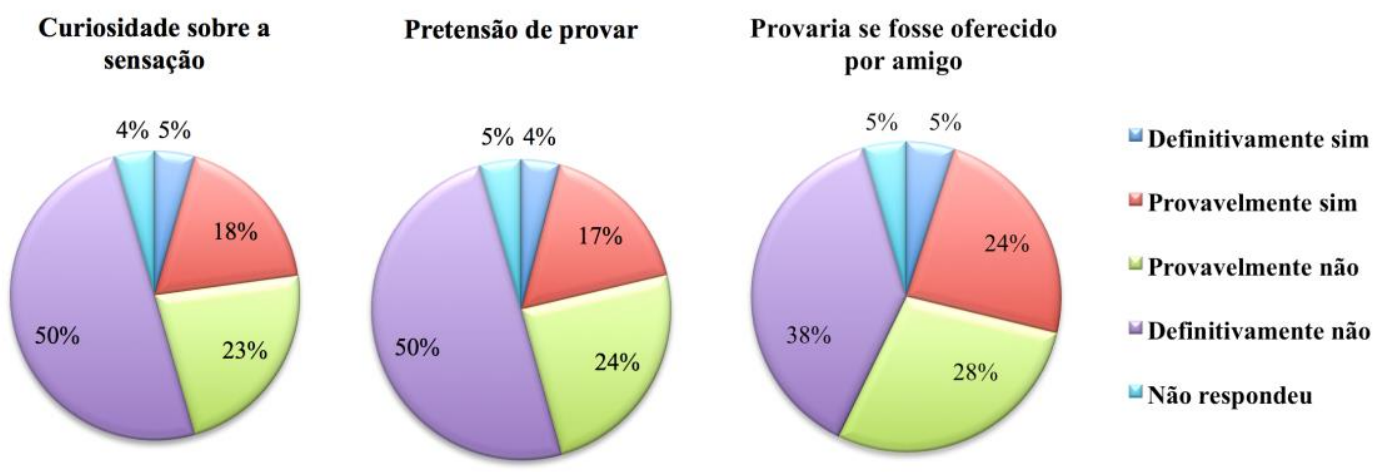

Figura 1. Porcentagem, entre os estudantes que declararam nunca ter utilizado cigarros eletrônicos, das respostas sobre curiosidade e pretensão de uso, bem como o impacto do fator social na decisão de provar o dispositivo

Com relação à autopercepção dos concluintes, 20,8\% dos estudantes afirmaram participantes quanto ao nível de não conhecer nada sobre o assunto, e 4,2\% conhecimento sobre CE (figura 2), nos três afirmou ter um alto nível de conhecimento. grupos a maioria considerou seu nível de Entre ingressantes e intermediários no curso, conhecimento como sendo baixo ( $\mathrm{G} 1=57,1 \%$, nenhum estudante declarou ter um alto nível G2 $=59,7 \%$ e G3=50\%). No grupo dos de conhecimento.

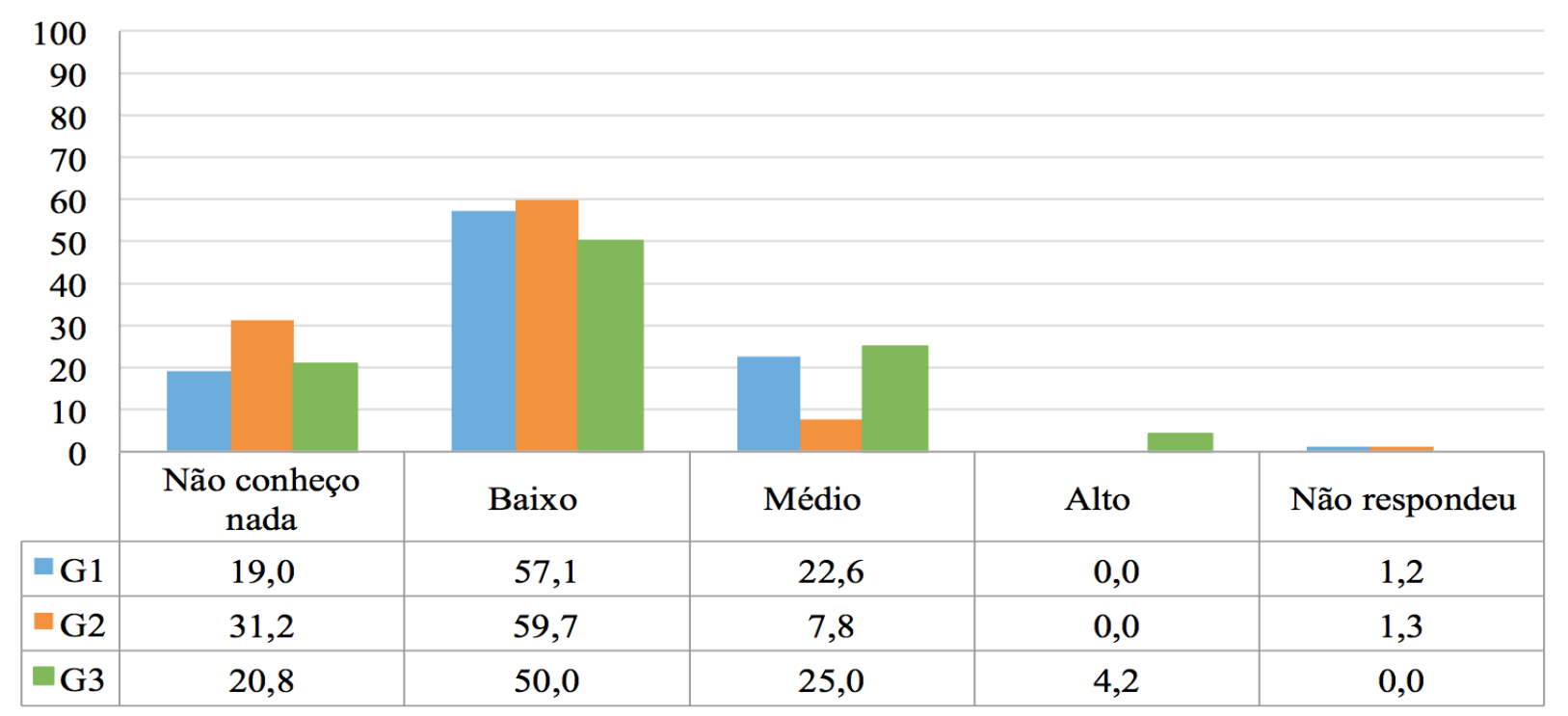

Figura 2. Porcentagem das respostas relativas à autopercepção sobre o nível de conhecimento sobre cigarros eletrônicos

Quando questionados sobre os riscos para a saúde do uso de $\mathrm{CE}$ em relação aos cigarros convencionais, a maioria dos estudantes acredita que CE são tão prejudiciais (média = 
$43,9 \%$ ) ou menos prejudiciais (média $=37,7 \%$ ) que os cigarros convencionais (figura 3 ).

O gráfico da figura 4 compila os dados referentes ao conhecimento dos participantes sobre os cigarros eletrônicos, considerando apenas as respostas corretas.

Com relação à afirmação de que seria permitido usar $\mathrm{CE}$ onde o cigarro convencional é proibido (locais fechados e públicos), $50 \%$ dos estudantes do G3 responderam corretamente que a afirmativa é falsa; nos grupos G1 e G2, $34,5 \%$ e $39 \%$ dos participantes identificaram a afirmativa como falsa.

Quando afirmado que o uso de CE pode ajudar as pessoas a parar de fumar cigarros convencionais, $\quad 47,9 \%$ dos concluintes identificaram corretamente a premissa como verdadeira, assim como $35,1 \%$ dos estudantes intermediários e $36,9 \%$ dos ingressantes.

A disponibilidade de saborizantes / aromatizantes de sabores agradáveis para uso nos CE foi corretamente identificada por $54,2 \%$ dos concluintes, $54,8 \%$ dos ingressantes $\mathrm{e}$
46,8\% dos intermediários.

No que se refere à afirmativa falsa de que o CE não geraria o chamado fumo passivo, $66,7 \%$ dos concluintes identificaram corretamente a questão como falsa, um resultado bem superior ao grupo dos ingressantes $(47,5 \%)$ e intermediários $(41,6 \%)$. Já na questão que afirmava que o uso de CE envolveria menores custos em relação aos cigarros convencionais (afirmativa considerada verdadeira com base em dados internacionais), foi observado o menor número de acertos ( $\mathrm{G} 1=$ $2,4 \%, \mathrm{G} 2=0 \%$ e $\mathrm{G} 3=4,2 \%$ ).

$\mathrm{Na}$ afirmativa falsa de que $\mathrm{CE}$ não possuiriam substâncias cancerígenas na sua composição, novamente o grupo dos concluintes obteve melhor desempenho, com $66,7 \%$ de acertos, contra $51,9 \%$ de acertos no G2 e 45,2\% no G1. Finalmente, frente à afirmativa verdadeira de que $\mathrm{CE}$ poderiam conter nicotina, $79,2 \%$ do G1 acertaram a questão, assim como $60,7 \%$ do G1 e $45,5 \%$ do $\mathrm{G} 2$.

\section{Cigarros eletrônicos são... em relação aos cigarros convencionais}

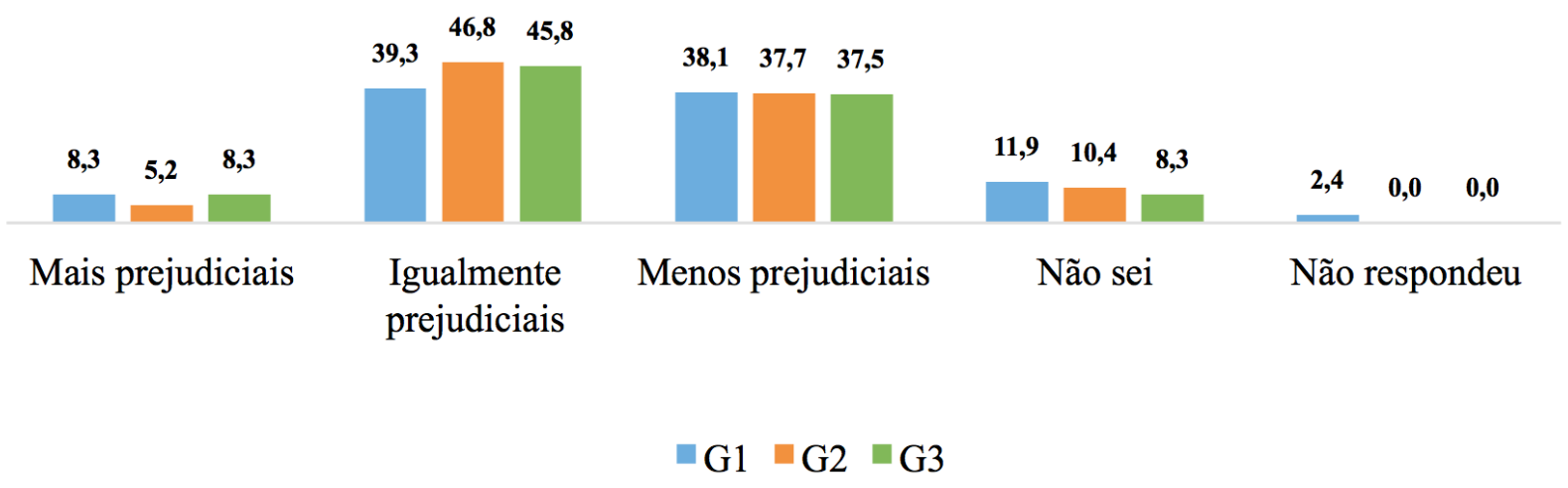

Figura 3. Porcentagem da percepção dos estudantes sobre os riscos para a saúde do uso de cigarros eletrônicos, comparativamente ao uso de cigarros convencionais 


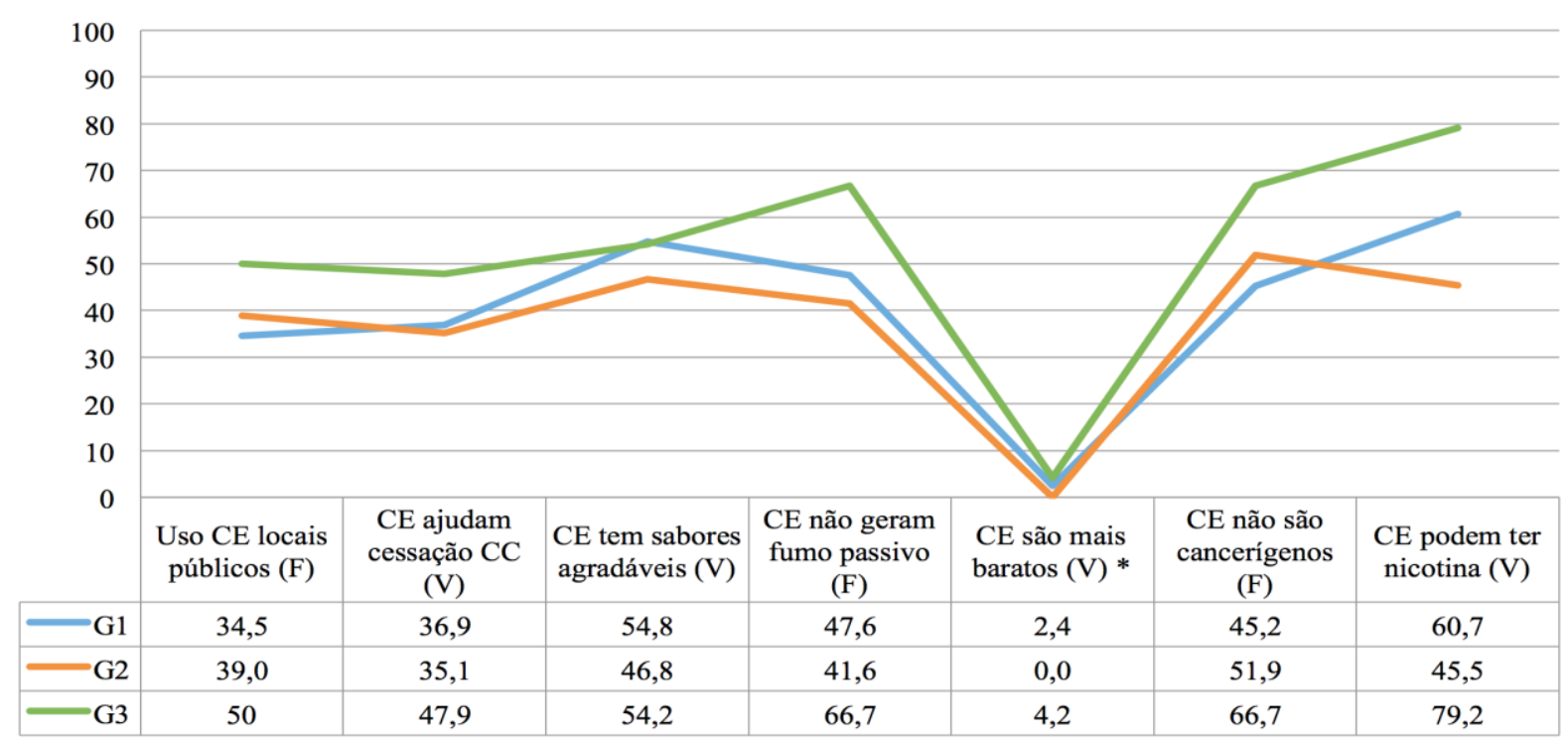

Figura 4. Porcentagem de acertos nos grupos G1, G2 e G3, das questões relacionadas a conhecimento sobre cigarros eletrônicos

Para permitir comparação entre o nível de uma análise visual comparativa desse resultado, conhecimento entre os grupos, foi feita a sendo possível observar que nos três grupos a categorização em conceitos A (considerado como maioria dos estudantes alcançou o conceito B. A conhecimento satisfatório, já que representa acima maior parcela de conceitos A foi obtida pelo grupo de $60 \%$ de acertos, com total de 7,6 e 5 acertos) e dos concluintes (39,5\%). A curva de B (considerado como conhecimento insatisfatório, conhecimento, considerando a categorização total de 4, 3, 2, 1 e 0 acertos). A figura 5 oferece proposta, foi ascendente.

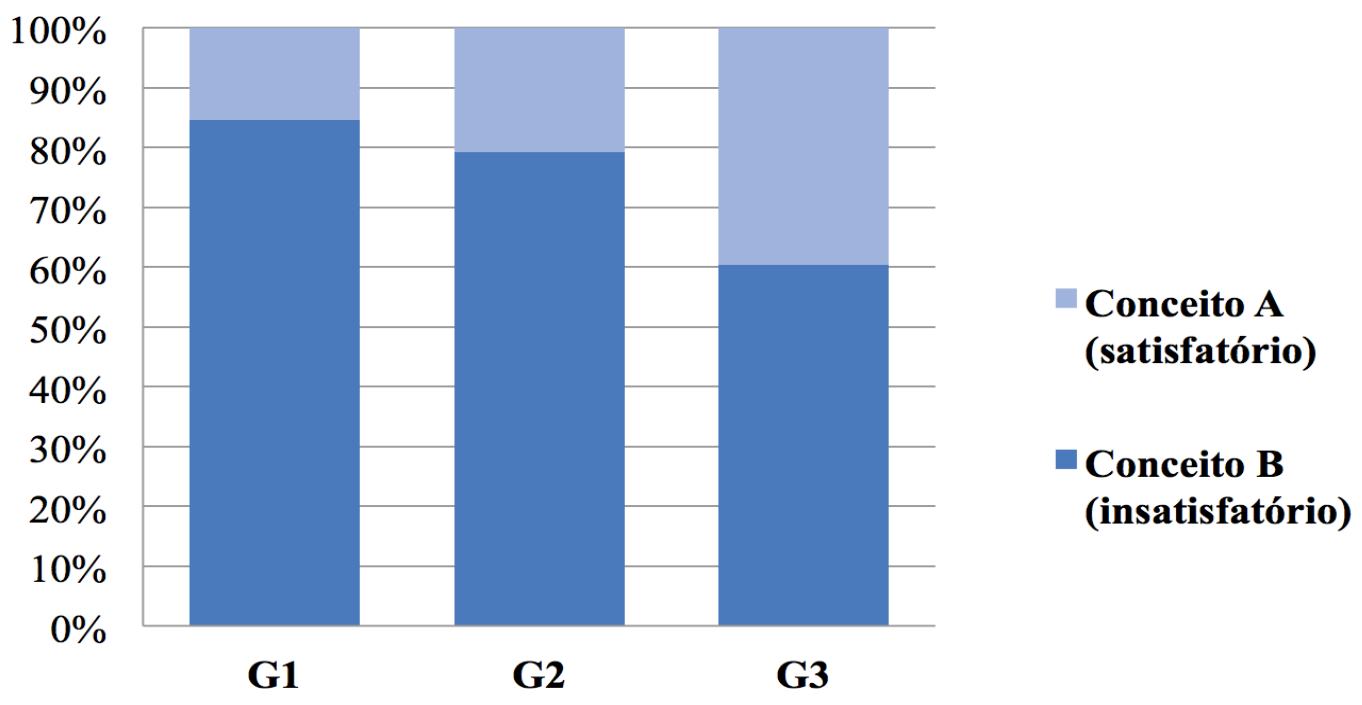

Figura 5. Comparativo dos conceitos obtidos pelos grupos no que se refere ao conhecimento sobre cigarros eletrônicos 
Para verificar se houve diferença estatisticamente significativa entre o nível de conhecimento de G1, G2 e G3, os dados foram submetidos ao teste do qui-quadrado. Não foi observada diferença estatisticamente significante entre o nível de conhecimento de G1 e G2 $(\mathrm{p}=0,382)$. Entretanto, diferença estatística foi observada entre o G1 e G3 ( $\mathrm{p}=0,002)$ e entre G2 e G3 ( $\mathrm{p}=0,023)$, mostrando que os estudantes concluintes possuem mais conhecimentos sobre o cigarro eletrônico que aqueles ingressantes ou intermediários.
Um dado interessante e que cabe ser destacado é que, visando evitar respostas aleatórias e permitir aos estudantes que informassem quando não sabiam a resposta, foi incluída a opção de resposta "não sei" nas questões relativas ao conhecimento sobre $\mathrm{CE}$. Para análise dos dados, essa opção foi incluída como resposta errada; no entanto, acreditamos ser necessário explicitar a porcentagem de participantes que admitiram não conhecer as respostas para as questões. O gráfico da figura 6 é representativo dessa situação.

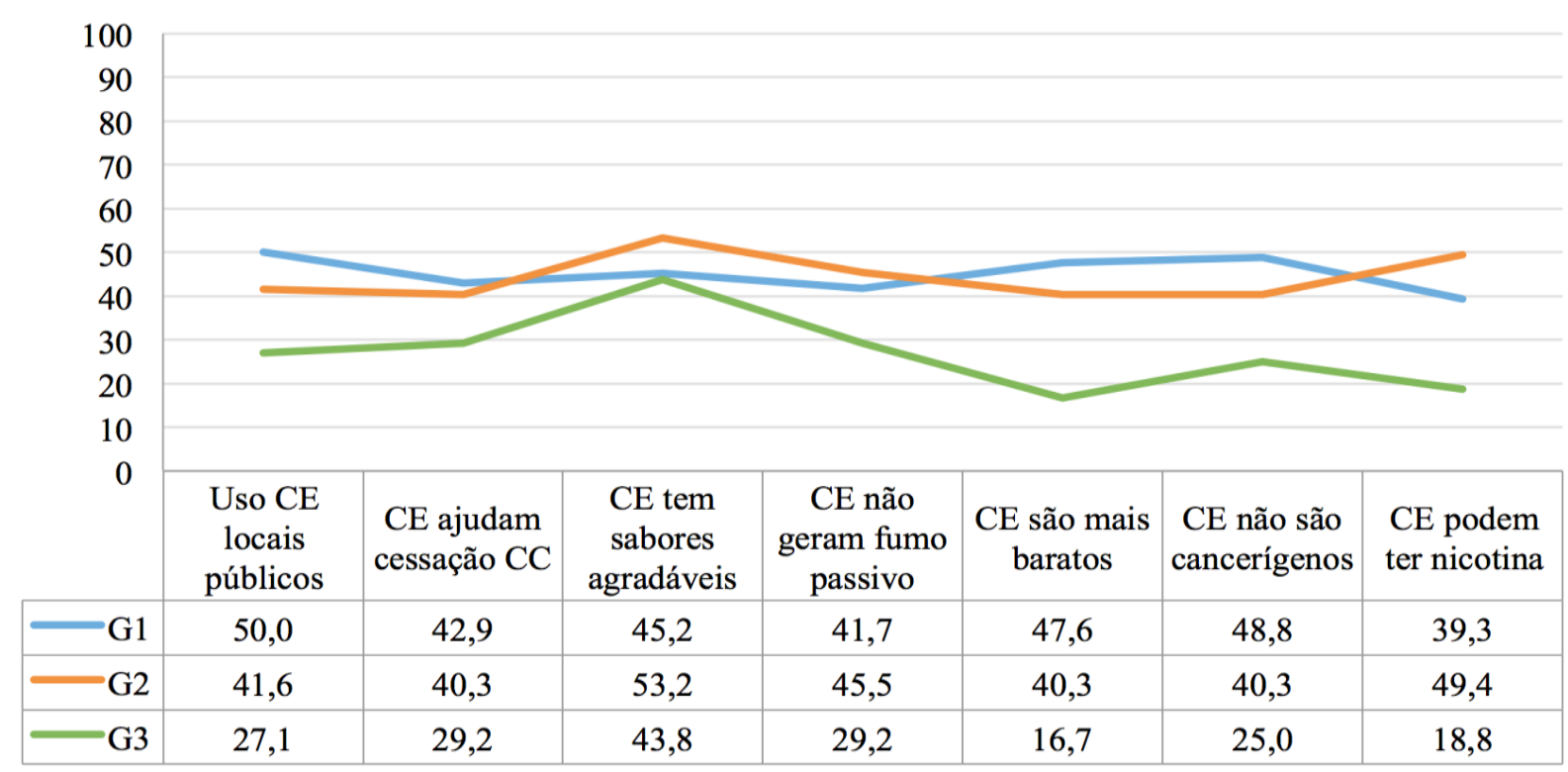

Figura 6. Porcentagem de estudantes que afirmou não conhecer a resposta correta, por grupo

No que diz respeito aos conhecimentos recebidos durante o Curso de Odontologia sobre CE (figura 7), foi possível constatar que em todos os grupos a ampla maioria dos participantes afirmou não ter recebido informações sobre os impactos do dispositivo na saúde ou de discutir sobre os motivos pelos quais as pessoas os utilizam.

Apesar disso, a grande maioria dos estudantes ( $100 \%$ no caso do G3) acredita que o cirurgião-dentista deve ter conhecimentos sobre o assunto, sendo que a maior parte se sente despreparada para orientar um paciente que lhe questione sobre o uso do CE (figura 8). 
Recebeu informações sobre os impactos dos cigarros eletrônicos na saúde?

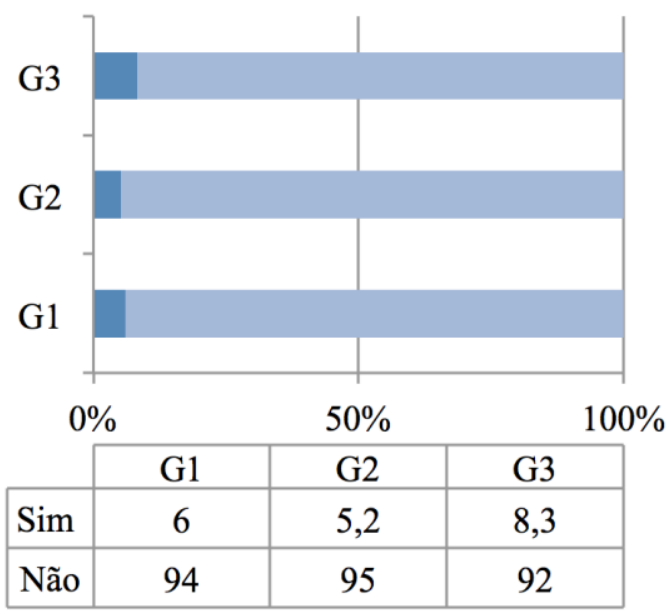

Teve oportunidade de discutir em sala os motivos das pessoas usarem cigarros eletrônicos?

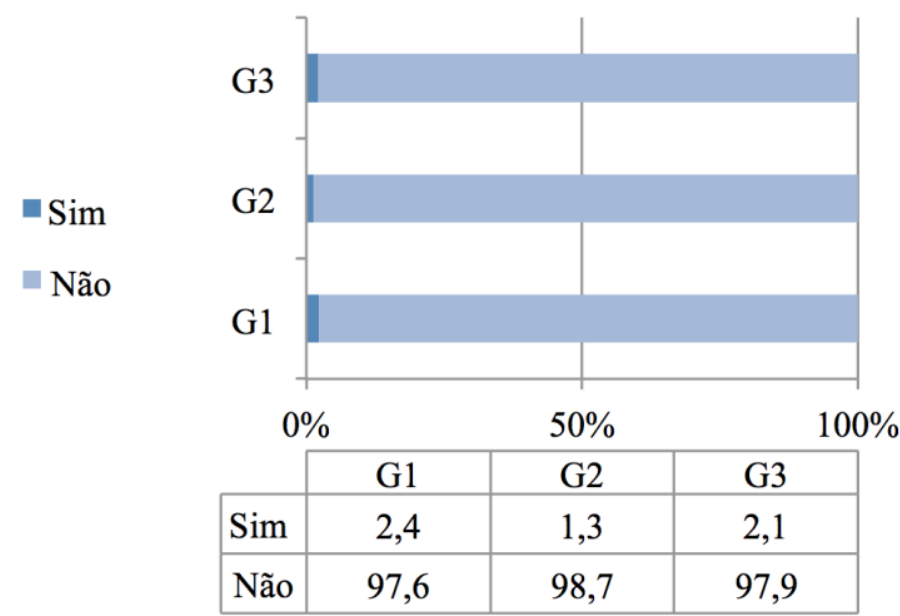

Figura 7. Conhecimentos recebidos durante o curso de Odontologia sobre cigarros eletrônicos

Opinião: é papel do CD ter conhecimentos sobre cigarros eletrônicos?

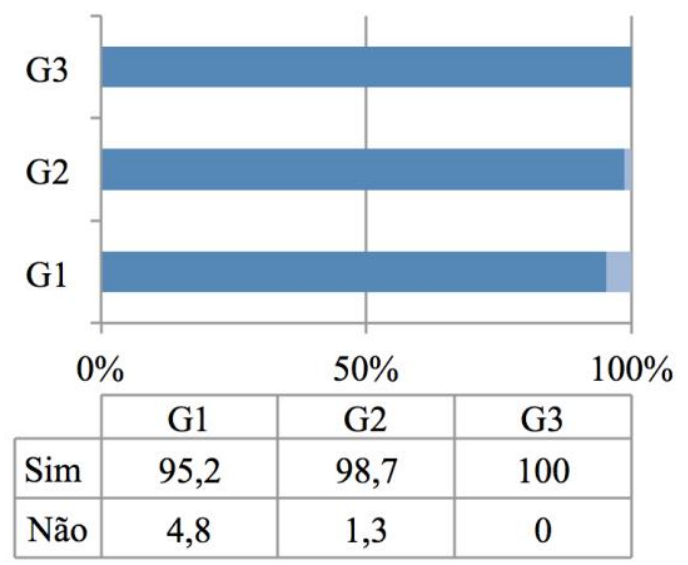

\section{Sente-se preparado para orientar um paciente que questione sobre cigarros eletrônicos?}

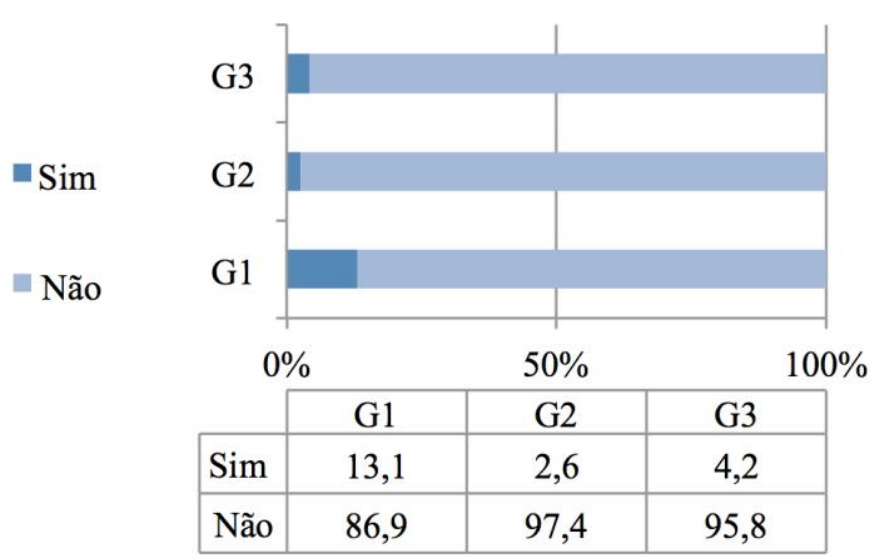

Figura 8. Opinião dos estudantes de Odontologia, segundo os grupos, sobre o papel do cirurgiãodentista e a percepção do preparo para orientar pacientes que questionem sobre cigarros eletrônicos

\section{DISCUSSÃO}

Dos 209 respondentes, apenas $11,76 \%$ afirmou ser ou ter sido usuário de cigarros eletrônicos, e a maioria se declarou não-tabagista $(85,3 \%)$. O G1 apresentou o maior número de autodeclarados tabagistas (19\%) ou ex-tabagistas $(8,3 \%)$, o que pode indicar que os estudantes iniciantes estão tendo mais contato com os produtos do tabaco do que aqueles que ingressaram no curso dois ou quatro anos antes. Merece 
referência o fato de 4,8\% dos respondentes do G1 terem optado por não responder ao questionamento sobre o status tabagista, o que pode indicar que se enquadram nessa categoria mas não se sentiram confortáveis para fornecer tal informação. Por outro lado, também deve ser considerada a possibilidade de subnotificação entre estudantes intermediários e concluintes, já que seria socialmente menos aceito que futuros profissionais da saúde façam uso de CE. Curiosamente, no grupo dos concluintes, $95,8 \%$ se autodeclarou não tabagista, mas $12,5 \%$ informou fazer ou ter feito uso de CE; essa inconsistência pode refletir a subnotificação do status tabagista ou ser explicada pelo fato de usuários de CE não se considerarem tabagistas, como citado na literatura ${ }^{12,21}$. Constatou-se ainda que em todos os grupos havia usuários de CE.

Entre os não usuários, a grande maioria afirmou saber da existência do dispositivo, e metade declarou que definitivamente não tem curiosidade ou pretensão de prová-lo, enquanto $23 \%$ declarou ter curiosidade e $21 \%$ ter pretensão de provar (provavelmente ou definitivamente). No entanto, quando questionados se provariam no caso de um dos melhores amigo(a)s oferecerem, 29\% responderam que sim ou provavelmente sim caindo para $38 \%$ aqueles que afirmaram que definitivamente não provariam. Estes achados sugerem que metade dos estudantes não estariam propensos a utilizar o equipamento, mas que a pressão social ou a confiança interpessoal poderia mudar a posição de cerca de $12 \%$ dos respondentes, o que também tem sido relatado na literatura ${ }^{8,22}$.

Quando questionados sobre os riscos para a saúde do uso de CE em relação aos cigarros convencionais, a maioria dos estudantes declarou acreditar que os CE são tão ou menos prejudiciais que os cigarros convencionais. Embora as opiniões dos autores se dividam quanto à segurança do seu uso, a literatura mostra o CE como uma opção mais segura à saúde quando comparada ao cigarro convencional $^{7,14,23,24}$; no entanto, ressaltam que os seus efeitos a longo prazo ainda não são conhecidos 16,17,24,25. Um editorial do Journal of American Dental Association alertou para a urgência no desenvolvimento de pesquisas para verificar os efeitos do CE na cavidade bucal, recomendando que os cirurgiões dentistas advertissem seus pacientes - entre eles os adolescentes - sobre riscos que ainda são desconhecidos, em face do aumento de 9 vezes no consumo de cigarros eletrônicos entre estudantes do ensino médio nos EUA ${ }^{25}$.

No conjunto das questões relativas ao conhecimento sobre $\mathrm{CE}$, os resultados mostraram que o número de acertos foi baixo, estando a grande maioria dos estudantes no nível considerado insuficiente - o que corrobora a análise autoperceptiva. O maior número de estudantes com conceito considerado satisfatório pertencia ao G3 (concluintes), mas mesmo nesse grupo apenas 39,5\% alcançou conceito A; assim, mesmo que a curva de conhecimento tenha sido ascendente (ou seja, que os alunos concluintes tenham demonstrado maior conhecimento que os de fases anteriores), o nível de conhecimento foi considerado muito baixo.

O desconhecimento da maioria dos estudantes de que o CE não pode ser usado em locais fechados ou públicos pode indicar que os mesmos estão usando (ou observando o seu uso) nesses ambientes, e/ou que desconhecem a proibição da utilização do dispositivo eletrônico no Brasil ${ }^{15}$.

De forma similar, os resultados mostraram que mais da metade dos estudantes em todos os grupos desconhece que os CE podem ajudar as pessoas a parar de fumar. No entanto, há algumas considerações a serem feitas nesse sentido. Devese explicitar que a cessação do tabagismo citada nos estudos é representada pela diminuição ou suspensão do uso do cigarro convencional - que frequentemente é simplesmente substituído justamente pelo uso do $\mathrm{CE}^{2,4,6,7}$. Pelo fato do CE 
não envolver o processo de combustão, ele vem sendo considerado mais seguro que o cigarro convencional $^{21,26}$. Para muitos autores, inclusive, o CE é comparável a outros dispositivos para reposição de nicotina (como os adesivos ou gomas de mascar), com a vantagem de simular as sensações sensoriais e motoras do ato de fumar ${ }^{5,14}$. Ressalta-se, no entanto, que as taxas de cessação do uso do cigarro convencional não são altas, variando de $7,3 \%$ a $26 \%$ e não se mantendo no longo prazo $2,4-7$.

Com relação à presença de sabores agradáveis nos $\mathrm{CE}$, os resultados mostraram que quase metade dos respondentes desconheciam essa possibilidade. Se por um lado isso pode confirmar que essa parcela dos participantes não teve contato com este perfil de equipamento, por outro lado diminui as chances de incluírem, em sua anamnese, perguntas envolvendo esse conhecimento. Em se tratando principalmente do atendimento em saúde prestado a adolescentes e jovens, é importante que os cirurgiões-dentistas estejam preparados para tal abordagem, já que a inclusão de sabores e aromas parece ser um grande atrativo para essa faixa etária da população se iniciar nessa prática ${ }^{8,9,11,13}$.

A literatura afirma que substâncias potencialmente nocivas podem ser encontradas no vapor gerado pelo CE ${ }^{13,27}$, podendo haver exposição involuntária de não usuários. Ao serem questionados sobre o fumo passivo gerado pelos cigarros eletrônicos, a maioria dos estudantes do G3 $(66,7 \%)$ identificaram corretamente que este é considerado um risco. Já no G1 e no G2, a taxa de acertos foi menor $(47,6 \%$ e $41,6 \%$, respectivamente). Isto pode indicar que os estudantes estão recebendo informações sobre fumo passivo no decorrer do curso; porém, o resultado ainda é bastante insatisfatório, uma vez que a quantidade de alunos concluintes que não souberam responder a esta questão foi maior que $30 \%$.

A questão sobre o custo dos $\mathrm{CE}$ em comparação com os cigarros convencionais foi a que resultou em menor número de acertos. Para a definição da resposta considerada correta nesta questão, foram utilizadas informações disponíveis em publicações da área nos EUA e na Europa, que dão conta que o custo anual é muito reduzido com o uso de CE, embora o custo inicial possa ser maior em função da compra do equipamento ${ }^{22,28}$. Como a venda de CE não é permitida no Brasil, optou-se por não efetuar essa comparação no mercado nacional (embora seja possível adquirir o equipamento em lojas do ramo); não havendo controle sobre a venda, também é possível que os custos no Brasil estejam acima do mercado internacional. Assim, deve-se considerar esse resultado com cautela, embora seja um ponto importante na escolha dos usuários pelos dispositivos, seja para diversão ou como alternativa para reposição de nicotina.

No que se refere à presença de substâncias cancerígenas no $\mathrm{CE}$, novamente a maior taxa de acertos foi no G3 $(66,7 \%)$, seguido pelo G2 $(51,9 \%)$ e G1 (45,2\%). De fato, estudos afirmam que o líquido aquecido pelo $\mathrm{CE}$ é composto de várias substâncias, algumas delas consideradas inertes para consumo por via oral, mas que quando aquecidas formam subprodutos com potencial carcinogênico ${ }^{13,14}$.

Quanto à possibilidade da presença de nicotina no dispositivo eletrônico, novamente os estudantes concluintes obtiveram maior número de acertos (79\%), seguidos pelos iniciantes $(60,7 \%)$ e intermediários (45,5\%); o maior número de acertos da questão no G1 pode ter relação com o fato deste grupo ter mais estudantes que fazem ou fizeram uso dos CE. A composição do líquido do CE pode ser bastante variada, e estudos indicam que a nicotina pode ou não estar presente; se estiver, pode ser apresentada em diferentes concentrações 3,13,26. Outra preocupação a respeito da nicotina é que a informação contida no rótulo do líquido do $\mathrm{CE}$ pode não ser precisa e/ou condizente com o 
conteúdo da solução; há relatos de embalagens rotuladas como "sem nicotina", e quando da análise do líquido a substância foi identificada em sua formulação ${ }^{29}$.

Finalmente, o fato de uma porcentagem muito pequena de estudantes terem recebido informações sobre os efeitos dos $\mathrm{CE}$, ou de não terem tido oportunidade de compreender os motivos pelos quais as pessoas utilizam esses equipamentos, é motivo de preocupação, considerando a escalada de utilização do dispositivo no mundo e, potencialmente, no Brasil, especialmente entre os jovens. Por outro lado, os estudantes parecem estar abertos a aprender sobre o assunto, entendendo que tal conteúdo deveria fazer parte da bagagem de conhecimentos do cirurgião-dentista. Considerando o nível de conhecimento levantado, é compreensível que os estudantes se sintam despreparados para orientar os seus pacientes; curiosamente, apesar do nível de conhecimento ter sido maior no grupo dos concluintes, este foi o grupo que declarou se sentir menos preparado para tal abordagem.

$\mathrm{Na}$ revisão realizada, foram encontrados apenas dois artigos levantando o conhecimento e atitudes de estudantes de Odontologia frente aos CE, com dados de Espanha e Estados Unidos ${ }^{30} \mathrm{e}$ da Arábia Saudita ${ }^{31}$. Apesar da dificuldade em comparar os resultados considerando as diferentes metodologias, ambos os estudos evidenciaram lacuna semelhante no conhecimento sobre o tema e a percepção dos estudantes de que não estão preparados para prover informações confiáveis aos pacientes.

Do conhecimento dos autores, este é o primeiro estudo que avalia o conhecimento sobre CE entre estudantes de Odontologia no Brasil. Uma limitação a ser considerada é a possibilidade de uma parcela dos estudantes não ter se sentido confortável para compartilhar hábitos e crenças, dada a sensibilidade do tema para a área da saúde. Apesar da amostra ser representativa do curso em questão, ela pode ser considerada pequena, sendo desejável que pesquisas futuras envolvam um maior número de estudantes de diferentes instituições de ensino, permitindo desenhar um perfil que seja representativo da situação nacional.

Considerando os resultados deste estudo, entende-se necessária a reflexão dos Núcleos Docentes Estruturantes sobre a pertinência de incluir atividades que permitam aos estudantes desenvolver competências para a abordagem e orientação ao paciente tabagista, contemplando também as novas formas de uso da nicotina. Ainda, e considerando o potencial aumento do uso dos cigarros eletrônicos por adolescentes e adultos jovens no Brasil, deve ser considerada a necessidade de incluir, na anamnese, perguntas que permitam identificar os seus usuários e orientá-los adequadamente quanto aos potenciais riscos desse hábito.

\section{CONCLUSÕES}

Os achados deste estudo fornecem um importante alerta sobre a necessidade de proporcionar aos estudantes oportunidades para desenvolver competências para a abordagem e orientação ao paciente tabagista contemplando as novas formas de uso da nicotina. Embora os estudantes tenham afirmado conhecer a existência dos CE e a curva de conhecimento ter sido ascendente, o nível de conhecimento foi considerado insatisfatório. Apesar de acreditarem que tal conhecimento deve fazer parte da formação de um cirurgião-dentista, a grande maioria dos estudantes declarou não ter recebido informações sobre CE durante o curso, não se sentindo preparados para responder a questionamentos de pacientes sobre o uso desses dispositivos.

\section{ABSTRACT \\ Level of knowledge of undergraduate dental students about electronic cigarettes}

The electronic cigarette has emerged as an 
alternative to the conventional cigarette, being considered by some as a smoking cessation aid. The device has attracted the interest of many young people in search of new experiences, sensations, and recreation. The aim of this study was to assess, using a questionnaire, the level of knowledge of undergraduate dental students from the Federal University of Santa Catarina (Florianópolis, Brazil) about electronic cigarettes. Information was also collected on the interest in trying the device, the approach of the subject in the course, and the self-perceived ability to inform patients about electronic cigarettes. The questionnaire was applied in April and May 2019 to 209 students over 18 years old, who were divided into 3 groups: freshmen (1st and 2nd semesters of the course), junior (4th and 5th semesters), and senior (9th and 10th semesters). The descriptive and statistical analyses were performed, and the significance level of $95 \%$ was adopted. The results showed that senior students had higher knowledge about electronic cigarettes than freshmen or junior students. However, the level of knowledge was considered unsatisfactory, as about $40 \%$ of the senior students had adequate level of knowledge (at least $60 \%$ of correct answers). Most students reported not having had contact with the topic during the undergraduate course. Likewise, most senior students reported not feeling prepared to advise patients on electronic cigarettes. The results of this study indicate the dental curriculum should include this topic in the training of future dentists, enabling them to inform their patients about the risks and benefits of using electronic cigarettes.

Descriptors: Electronic Nicotine Delivery Systems. Knowledge. Education, Dental.

\section{REFERENCIAS}

1. Pinto MT, Pichon-Riviere A, Bardach A. Estimativa da carga do tabagismo no Brasil: mortalidade, morbidade e custos. Cad Saude Publica. 2015 Jun;31(6):1283-97.

2. Manzoli L, La Vecchia C, Flacco ME, Capasso L, Simonetti V, Boccia S, et al. Multicentric cohort study on the long-term efficacy and safety of electronic cigarettes: study design and methodology. BMC Public Health. 2013 Dec 24;13(1):883.

3. Hartmann-Boyce J, McRobbie H, Bullen C, Begh R, Stead LF, Hajek P. Electronic cigarettes for smoking cessation. Cochrane Database Syst Rev. $2016 \quad$ Sep 13;2016(9):178-9.

4. Adriaens K, Van Gucht D, Declerck P, Baeyens F. Effectiveness of the electronic cigarette: an eight-week flemish study with six-month follow-up on smoking reduction, craving and experienced benefits and complaints. Int J Environ Res Public Health. 2014 Oct 29;11(11):11220-48.

5. Bullen C, Howe C, Laugesen M, McRobbie $\mathrm{H}$, Parag V, Williman J, et al. Electronic cigarettes for smoking cessation: a randomised controlled trial. Lancet. 2013 Nov;382(9905):1629-37.

6. Caponnetto P, Campagna D, Cibella F, Morjaria JB, Caruso M, Russo C, et al. EffiCiency and Safety of an eLectronic cigAreTte (ECLAT) as tobacco cigarettes substitute: a prospective 12-month randomized control design study. PLoS One. 2013 Jun 24;8(6):e66317.

7. Hajek P, Phillips-Waller A, Przulj D, Pesola $\mathrm{F}$, Myers Smith $\mathrm{K}$, Bisal $\mathrm{N}$, et al. A randomized trial of e-cigarettes versus nicotine-replacement therapy. N Engl J Med. 2019 Feb 14;380(7):629-37.

8. Roditis M, Delucchi K, Cash D, HalpernFelsher B. Adolescents' perceptions of health risks, social risks, and benefits differ across tobacco products. J Adolesc Heal. 2016/04/25. 2016 May;58(5):558-66.

9. Walley SC, Wilson KM, Winickoff JP, Groner J. A public health crisis: electronic cigarettes, vape, and JUUL. Pediatrics. 2019 Jun 23;143(6):e20182741.

10. Willett JG, Bennett M, Hair EC, Xiao H, 
Greenberg MS, Harvey E, et al. Recognition, use and perceptions of JUUL among youth and young adults. Tob Control. 2018 Apr 18; tobaccocontrol-2018-054273.

11. Krishnan-Sarin S, Jackson A, Morean M, Kong G, Bold KW, Camenga DR, et al. Ecigarette devices used by high-school youth. Drug Alcohol Depend. 2019 Jan;194:395400.

12. INCA - Instituto Nacional de Câncer. Cigarros eletrônicos: o que sabemos? Estudo sobre a composição do vapor e danos à saúde, o papel na redução de danos e no tratamento da dependência de nicotina. Rio de Janeiro; 2016. 120 p.

13. Jimenez Ruiz CA, Solano Reina S, de Granda Orive JI, Signes-Costa Minaya J, de Higes Martinez E, Riesco Miranda JA, et al. El cigarrillo electrónico. Declaración oficial de la Sociedad Española de Neumología y Cirugía Torácica (SEPAR) sobre la eficacia, seguridad y regulación de los cigarrillos electrónicos. Arch Bronconeumol. 2014/04/02. 2014 Aug;50(8):362-7.

14. Sultan AS, Jessri M, Farah CS. Electronic nicotine delivery systems: Oral health implications and oral cancer risk. J Oral Pathol Med. 2018 Dec 14;jop.12810.

15. ANVISA - Agência Nacional de Vigilância Sanitária. Resolução RDC No. 46, de 28 de agosto de 2009 [Internet]. BRAZIL; 2009 p. 2. [Acesso em: 8 de março de 2021]. Disponível em: http://bvsms.saude.gov.br/ bvs/saudelegis/anvisa/2009/res0046_28_08 2009.html.

16. Cavalcante TM, Szklo AS, Perez C de A, Thrasher JF, Szklo M, Ouimet J, et al. Conhecimento e uso de cigarros eletrônicos e percepção de risco no Brasil: resultados de um país com requisitos regulatórios rígidos. Cad Saude Publica. 2017/09/28. 2017 Sep 21;33(suppl 3):e00074416.
17. Phillips JA. Electronic cigarettes: health risks and workplace policy. workplace health saf. 2014/09/11. 2014 Sep;62(9):396396.

18. Perrine CG, Pickens CM, Boehmer TK, King BA, Jones CM, DeSisto CL, et al. Characteristics of a multistate outbreak of lung injury associated with E-cigarette Use, or vaping - United States, 2019. MMWR Morb Mortal Wkly Rep. 2019 Oct 4;68(39):860-4.

19. Vaught B, Spellman J, Shah A, Stewart A, Mullin D. Facial trauma caused by electronic cigarette explosion. Ear Nose Throat J. 2017/03/28. 2017 Mar;96(3):139-42.

20. Villanti AC, Rath JM, Williams VF, Pearson JL, Richardson A, Abrams DB, et al. Impact of Exposure to Electronic Cigarette Advertising on Susceptibility and Trial of Electronic cigarettes and cigarettes in US young adults: a randomized controlled trial. Nicotine Tob Res. 2015/11/18. 2016 May;18(5):1331-9.

21. Caponnetto P, Campagna D, Papale G, Russo C, Polosa R. The emerging phenomenon of electronic cigarettes. Expert Rev Respir Med. 2012 Feb 9;6(1):63-74.

22. Directorate-General for Communication (European Commission). Attitudes of Europeans towards tobacco and electronic cigarettes [Internet]. European Union; 2017. [Acesso em: 08 de março de 2021]. Disponível em: https://op.europa.eu/en/publi cation-detail/-/publi cation/2f01a3d1-0af211e8-966a-01aa75ed71a1.

23. Goniewicz ML, Knysak J, Gawron M, Kosmider L, Sobczak A, Kurek J, et al. Levels of selected carcinogens and toxicants in vapour from electronic cigarettes. Tob Control. 2014 Mar;23(2):133-9.

24. MacDonald A, Middlekauff HR. Electronic cigarettes and cardiovascular health: what do 
we know so far? Vasc Health Risk Manag. 2019 Jun; 15:159-74.

25. Tomar SL, Fox CH, Connolly GN. Electronic cigarettes. The tobacco industry's latest threat to oral health? J Am Dent Assoc. 2015/09/01. 2015 Sep;146(9):651-3.

26. Farsalinos K, Romagna G, Tsiapras D, Kyrzopoulos S, Voudris V. Evaluation of Electronic Cigarette use (vaping) topography and estimation of liquid consumption: implications for research protocol standards definition and for public health authorities' regulation. Int J Environ Res Public Health. 2013 Jun 18;10(6):250014.

27. Czogala J, Goniewicz ML, Fidelus B, Zielinska-Danch W, Travers MJ, Sobczak A. Secondhand exposure to vapors from Electronic Cigarettes. Nicotine Tob Res. 2014 Jun;16(6):655-62.

28. Prochnow JA. E-cigarettes: A practical, evidence-based guide for advanced practice nurses. J Nurse Pract. 2017 Jul;13(7):44955.
29. National Centre for Smoking Cessation and Training (NCSCT). Eletronic cigarettes [Internet]. London (UK): National Centre for Smoking Cessation and Training (NCSCT); 2014. p. 16. [Acesso em: 18 de novembro de 2019]. Disponível em: https://www.ncsct. co.uk/.

30. Martín Carreras-Presas C, Naeim M, Hsiou D, Somacarrera Pérez ML, Messadi D V. The need to educate future dental professionals on E-cigarette effects. Eur $\mathrm{J}$ Dent Educ. 2018 Nov;22(4):e751-8.

31. Natto ZS. Dental Students' Knowledge and attitudes about Electronic Cigarettes: a cross-sectional study at one Saudi University. J Dent Educ. 2019;83(10):37-9.

\section{Correspondência para:}

Maria Inês Meurer

e-mail: meurer.m.i@ufsc.br

Universidade Federal de Santa Catarina,

Departamento de Patologia

Campus Reitor João David Ferreira Lima 88040-370 Florianópolis/SC 